\title{
Visual size difference discrimination: Effect of disc size and retinal locus'
}

\author{
M. L. MATTHEWS 2 \\ UNIVERSITY OF GUELPH
}

\begin{abstract}
Ability to discriminate between the sizes of two adjacently presented discs of light is considered both as a function of the size of the discs and the retinal locus of their presentation. Ss were required to report whether a comparison disc was seen as larger or the same size as a standard disc when the two were presented briefly together. Results showed that the ind required for $50 \%$ difference discrimination was independent of the size of the disc and was constant at about $30 \mathrm{sec}$ of arc. For peripheral presentations, the ind was found to increase in a linear manner with increasing eccentricity of presentation. Comparisons are drawn between the results obtained and peripheral visual acuity data and it is speculated that similar processes are involved in both situations.
\end{abstract}

While there is abundant information on the performance of the human eye and visual system with respect to detecting the presence or absence of light, discriminating between intensities, area/intensity and time/intensity relationships, relatively little is known about visual performance in a size discrimination situation. The question that might be asked is how effective is the eye in discriminating size differences between two objects of similar shape with respect to such stimulus parameters as length, width, or, in the case of circles, diameter. Although such situations have received relatively little attention, it is possible to have a priori expectations of visual performance. There appear to be two immediate hypotheses worth considering: (1) Difference discrimination might be considered a function of the size or magnitude of the stimulus, i.e., it would follow an approximation to Weber's law or one of its later derivatives. (2) We may consider that such discrimination could be thought of as a form of acuity task, in which case the jnd would be constant irrespective of stimulus size.

There is evidence to suggest that data can be found to support either of the above mentioned hypotheses depending upon the exact experimental conditions. Ono (1967) demonstrated that discrimination of the difference between the length of two lines gave a Weber-type relationship only when the two lines were spatially separated, thus forcing the $S$ to make a temporal judgment by first looking at one line and then the other. If simultaneous comparison was permitted with the two lines close together, the jnd was found to be approximately constant within experimental conditions and between three-line lengths. Ono points out that Weber specified that the relationship between the jnd and the size of the stimulus was for nonsimultaneous comparison only. Thus, experimental situations that permit a nonsimultaneous comparison between stimuli, whether in time or by permitting eye movements so that $\mathrm{S}$ is able to look from one stimulus to the other, are likely to tell us more about short-term visual storage than immediate visual performance with respect to the size-difference discrimination of discs of light in a situation that permits simultaneous comparison only.

In order to determine the validity of the analogy between visual size-difference discrimination and acuity, it is first necessary to establish whether the jnd between two discs of light is independent of the size of the discs. If the jnd is found to be constant, a second issue that should receive consideration is whether or not the jnd will increase as a function of the displacement of the discrimination task off the visual axis, since it is well established that visual acuity becomes progressively worse with increasing retinal eccentricity. The experiments described below are designed to give answers to these two specific issues.

\section{METHOD}

The description of the experimental method will be divided into two sections-first, dealing with size discrimination of discs in central vision as a function of the size of the discs, and second, size discrimination of discs in peripheral retinal regions.

\section{Size Discrimination in Central Vision}

The $S$ looked straight ahead with the preferred eye at a small fixation mark at eye level (the $S$ was seated with his head position maintained by clamps to the forehead and a chin rest) in the center of a large uniformly illuminated screen whose luminance was $1.05 \log \mathrm{mL}$. Two discs of light (luminance $1.85 \log \mathrm{mL}$ ) could be flashed simultaneously on the screen, one on either side of the fixation mark. Each disc of light was produced by transillumination of a precision-drilled hole in the center of a piece of mild steel machined to be mounted and stacked in a modified automatic slide change projector. Each disc was presented by an independent projector system with matched light sources. Each projector was equipped with a camera-type shutter to permit the delivery of short-duration flashes. In all experiments an indicated shutter speed of $125 \mathrm{msec}$ was used; calibration of the shutters revealed that exposures fell in a range of $130-133 \mathrm{msec}$. The two shutters could be triggered simultaneously by $\mathrm{E}$ at a central control point. Five disc sizes were used, 5, 12, 20,30, and $50 \mathrm{~min}$ of arc in angular diam when viewed by the $S$ at a distance of $28 \mathrm{ft}$ from the screen. The discs were projected with an angular separation of $1.5 \mathrm{deg}$ between their centers. In summary, two discs of light of equal luminance could be presented simultaneously on either side of the fixation mark for a short, constant duration.

Three Ss were used in the experiment; each was given the instruction that he was to detect the larger of two discs, given that in any experimental run the disc on one side of the fixation mark would always be the same size (standard disc--SD). The Ss were further instructed that the other disc (comparison disc-CD) would sometimes be larger than the SD and sometimes the same size. The two responses open to the Ss were "equal" and "larger." Step sizes between the CD and SD diameters could be as small as $0.1 \mathrm{~min}$ of arc. The CDs were presented in a random order in preference to a method of limits; SCs of the same size as the SD were also presented in order to determine guessing rates. On successive runs (a run usually consisted of 25-30 separate discriminations) the position of the SD was changed to the other side of the fixation mark in order to eliminate any positional effects that might occur with repeated presentation of the SD to one side of the visual field only.

\section{Size Discrimination in Peripheral Vision}

The viewing conditions in this experiment were the same as for central vision, but in this case the two discs were presented to the 


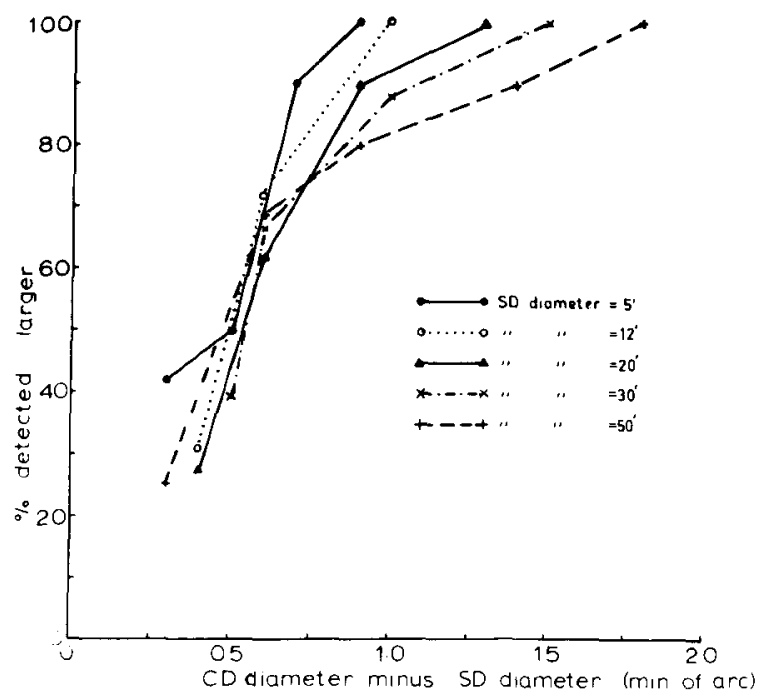

Fig. 1. Size difference discrimination by one $S$ (RS) for each of the comparison dises.

right of the fixation mark at varying eccentricities. Means of presentation, exposure duration, luminance, and psychophysical method were all the same as for the previous experiment. The discs were again presented with a separation of $1.5 \mathrm{deg}$ between their centers; the 112-, 30-, and 50-min discs only were used. The eccentricities of presentation were $6,12,18,24$, and $30 \mathrm{deg}$ from the fixation mark (eccentricity was measured from the fixation mark to the point between the discs equidistant from each of their centers). A beamsplitter system enabled $\mathrm{E}$ to monitor both the S's eye movements and the fixation mark so that presentations were only given when $S$ was accurately fixating. Three Ss took part in the experiment.

\section{RESULTS}

Figure 1 shows the results for $\mathrm{RS}$ in the central vision discrimination experiment; percentage-detected larger responses are plotted as a function of the size difference $(\Delta d)$ between the diameters of the SD and the CD in minutes of arc; each of the target sizes has been plotted on the same graph for comparison. Detection thresholds of $50 \%$ and $95 \%$ have been extracted from these results and are shown together with similar thresholds for $\mathrm{DP}$ and $\mathrm{JA}$ in Fig. 2 . In this case, $\Delta \mathrm{d}$ has been plotted as a function of the diameter of the SD. All three Ss give very similar results; the jnd for $50 \%$ is virtually constant for all disc sizes and shows good agreement between Ss. There is a marked trend for a greater response variability with larger target sizes as indicated by the $95 \%$ threshold values. The overall results show that size discrimination between discs is constant at about $0.5 \mathrm{~min}$ difference in diametric size whatever the size of the discs to be discriminated.

Figure 3 shows the results of Experiment 2 on peripheral discrimination. For each $\mathrm{S}, \Delta \mathrm{d}(50 \%$ detection) has been plotted as a function of eccentricity of presentation of the discs in degrees for the three disc sizes that were used. The nature of the relationship between discrimination and eccentricity seems to be linear for each $S$, although the slope values are a little different between Ss. There is no indication of any tendency for discrimination to be dependent on disc size. A least squares fit has been performed for each disc size and all three Ss taken together; this is shown in Fig. 4, where the coordinates are the same as in Fig. 3. The variability of the overall data is indicated by a plot of one standard deviation on either side of the mean. In this case, variability of response is found to increase with distance of presentation from the fixation mark. For comparison purposes, minimum angle of resolution derived from peripheral visual acuity data from Westheimer (1967) has also been presented and shows good agreement in both slope and intercept value with the size discrimination function.

In both experiments false alarm rates were very low and did not change significantly between the experimental conditions. Each data point is based upon 40 discriminations.
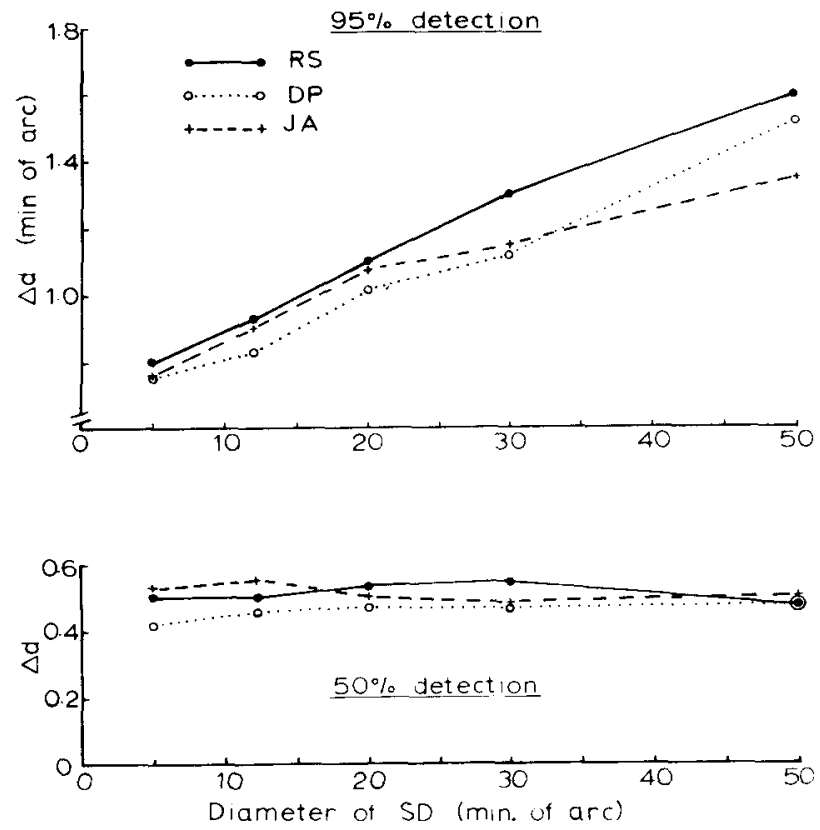

Fig. 2. Size difference discrimination for all three Ss at indicated detection rates of 'larger' responses. $\Delta \mathrm{d}$ is the difference in diameter between the CD and SD.
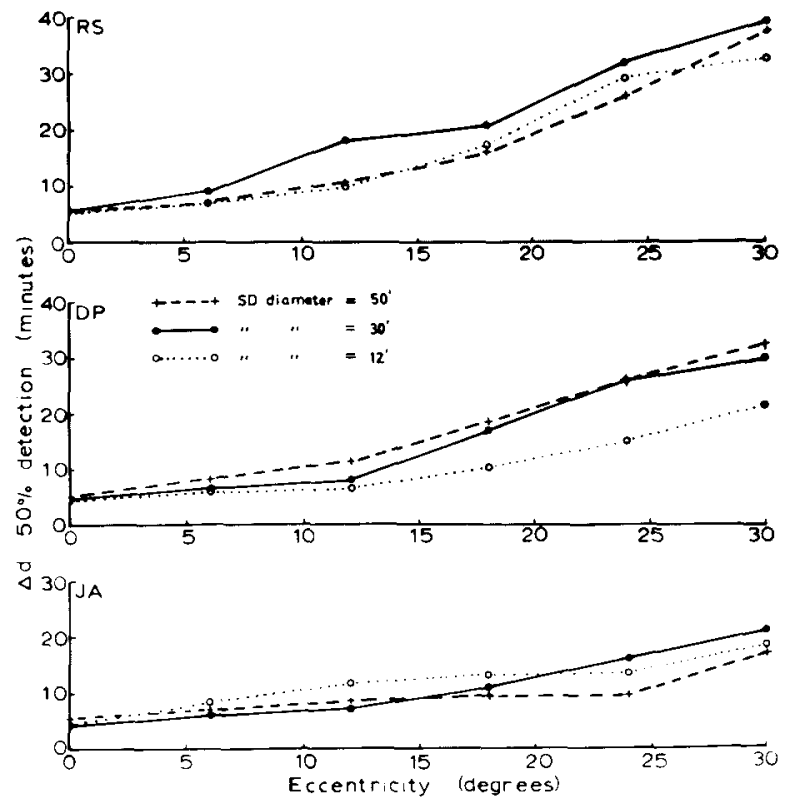

Fig. 3. Size difference discrimination $(50 \%)$ as a function of eccentricity of presentation of discs; each SD is indicated for each of three Ss. 


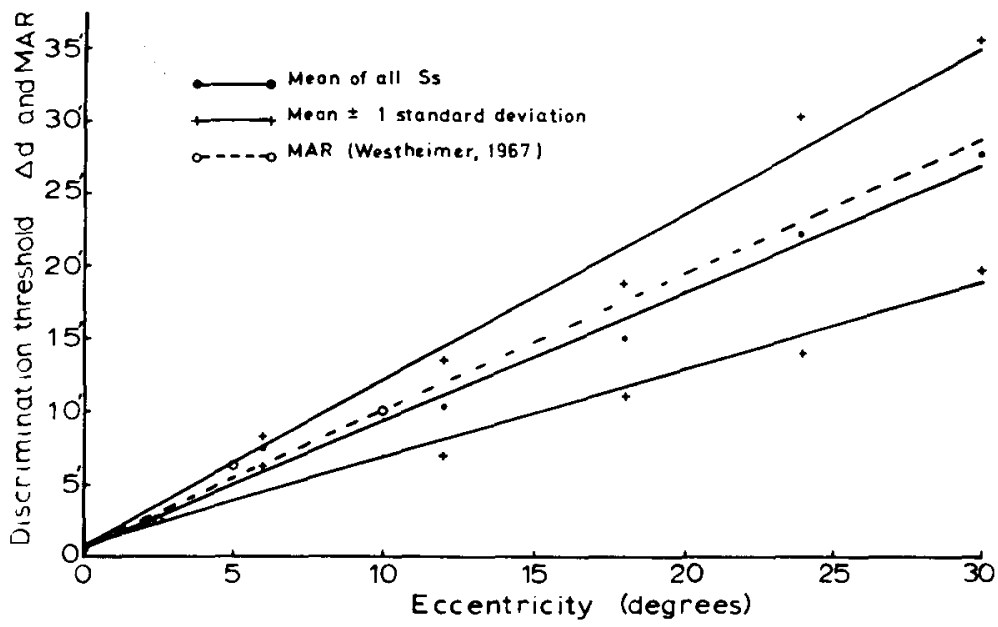

Fig. 4. Data for each $S$ and each SD averaged from Fig. 3. Data scatter is indicated by a plot of one standard deviation on either side of the mean. Minimum angle of resolution (derived from Westheimer, 1967) is shown on same coordinates for comparison. All data have been fitted by least squares regression lines.

\section{DISCUSSION}

In considering the results of both experiments, two immediate points become clear: (1) Size-difference discrimination is unaffected by the size of the test stimuli in a range from 5 to 50 min. (2) Ability to discriminate a size difference decreases in a linear manner with eccentricity of presentation. Both of these findings would suggest that the underlying processes involved in size-difference discrimination are essentially similar to those in visual acuity tasks. To find evidence to support this assertion we may look at data from similar situations in which comparable measurements have been made of visual acuity. It is a reasonable assumption that the kind of acuity task that most closely parallels size-difference discrimination is the recognition of the orientation of the gap in a Landolt C. Using data from Shlaer (1937), the minimum angle of resolution (the reciprocal of visual acuity) for the gap in a Landolt $\mathbf{C}$ for central vision at a luminance equivalent to that used in the present experiments is found to be approximately $0.45 \mathrm{~min}$, which corresponds closely to the mean of $0.48 \mathrm{~min}$ obtained in the present experiment from all $\mathrm{Ss}$ for all stimulus sizes $(\mathrm{SD}=0.036 \mathrm{~min})$. For peripheral vision the data in Fig. 4 that are taken from Westheimer (1967) show close agreement with the regression line from the present experiments; it should be noted that Westheimer's data were obtained from eccentricities less than $10 \mathrm{deg}$; the acuity task in this case was again gap detection in a Landolt C. Weymouth (1958) has pointed out that functions that are linear with eccentricity are typically found for all types of acuity tests; he shows that intercept values go from $0.43 \mathrm{~min}$ for vernier acuity to $4.6 \mathrm{~min}$ for one $\mathrm{S}$ for the Landolt $\mathrm{C}$ (figures are again minimum angle of resolution). Slope values were found to vary from about 0.11 for motion acuity to 1.80 for the Landolt $C$. The intercept and slope values from the present experiments were 0.6 and 0.88 , respectively, which fall within the error range of Weymouth's peripheral visual acuity data for 20 Ss on a Landolt $C$ task.

These findings would suggest that the size-difference discrimination ability is dependent upon the mechanisms that underlie other tasks in which spatial interactions are important. Weymouth considered that Panum area was the important neurophysiological correlate that might determine spatial resolution, since this too was found to increase in a manner that was linear with eccentricity. Much of the recent electrophysiological evidence, on the other hand, would suggest that spatial interactions are closely associated with organized receptive fields with familiar excitatory/inhibitory components. Although there is little direct electrophysiological evidence for a systematic linear variation in size of receptive field with eccentricity from the fovea, Hubel and Wiesel (1960) have shown that there is a general increase in the size of receptive fields recorded from retinal ganglion cells of the monkey as the stimulus is moved further into peripheral regions; Sterling and Wickelgren (1969) have produced similar findings from recordings in the superior colliculus of the cat. In both of these cases it would be possible to fit a linear relationship but the scatter of the data is so great that such an operation would have little consequence or meaning.

At a behavioral level, Westheimer (1967) found that the diameter of what might be considered a functional receptive field was closely related to visual acuity values for eccentricities up to 10 deg. In turn, it has been shown that Westheimer's visual acuity data are very close to the size-discrimination limits determined by the present experiments. Without any comparable evidence of receptive field sizes determined both by electrophysiological methods and by measuring peripheral visual acuity in the same organism, it would appear that all that may be stated in conclusion is that a similar type of spatial interaction underlies both tasks that assess visual acuity and size-difference discrimination ability. The smallest "unit" found for both types of situation is approximately $30 \mathrm{sec}$ in the fovea, which increases linearly to about $28 \mathrm{~min}$ at an eccentricity of $30 \mathrm{deg}$.

\section{REFERENCES}

HUBEL, D. H., \& WIESEL, T. N. Receptive fields of optic nerve fibers in the spider monkey. Journal of Physiology, 1960, 154, 572-580.

ONO, H. Difference threshold for stimulus length under simultaneous and nonsimultaneous viewing conditions. Perception \& Psychophysics, 1967, 2, 201-207.

SHLAER, S. The relation between visual acuity and illumination. Journal of General Physiology, 1937, 21, 165-188.

STERLING, P., \& WICKELGREN, B. G. Visual receptive fields in the superior colliculus of the cat. Journal of Neurophysiology, 1969, 32, $1-15$.

WESTHEIMER, G. Spatial interaction in human cone vision. Journal of Phy siology, 1967, 190, 139-154.

WEYMOUTH, F. W. Visual sensory units and the minimum angle of resolution. American Journal of Oph thalmology, 1958, 46, 102-113.

\section{NOTES}

1. This research was originally carried out under Ministry of Technology Contract No. PD/24/018/AT, awarded to Professor C. I. Howarth at the Department of Psychology, University of Nottingham, England. Additional support was made available at the author's present address from NRC Grant No. AP316.

2. Address: Department of Psychology, University of Guelph, Guelph, Ontario, Canada.

(Accepted for publication February 23, 1969.) 